Continued efforts to improve our practice and patient's adherence is essential.

Disclosure No significant relationships.

\section{P209 PRODUCTIVE IMPACT OF ASSISTED REFERRALS AND INCENTIVIZED ENROLLMENT ON THE UPTAKE OF HIV SERVICES IN LAGOS STATE, NIGERIA}

Felix Iwuala*. Association for Reproductive and Family Health (ARFH), Orphans and Vulnerable Children (OVC) Program, Lagos., Nigeria

\subsection{6/sextrans-2019-sti.356}

Background Nigeria has the second highest global prevalence of Human Immunodeficiency Virus (HIV), with over two million children (0-17 years) made vulnerable by HIV, having lost either or both parents to Acquired Immune Deficiency Syndrome (AIDS). The Association for Reproductive and Family Health (ARFH) is implementing a five year Local Partners for Orphans and Vulnerable Children (OVC) Project in Nigeria, with support from the United States Agency for International Development (USAID), to mitigate the impact of HIV/AIDS on children and vulnerable households, in Lagos State. Poverty remains a major driver of HIV in Nigeria.

Methods Strategies include HIV Risk Assessments, Assisted Referrals and incentivized enrollment. The Community Volunteers (CVs) accompany those referred for HIV Testing Services (HTS) to health facilities, results are collected and HIV positives are counselled and linked to treatment same day. Incentivized enrollment on treatment is for indigent enrollees, to promote retention. The sum of $\$ 25$ is provided in three equal instalments as coupons, redeemed on producing evidence of enrollment on treatment, and two subsequent drug refills.

Results All the 43,495 enrollees (males: 16,908, females: 26,587) know their HIV status. The data subsets include 31,396 OVC (males: 15,482, females: 15,914) and 12,099 Caregivers (males: 1,426, females: 10,673). Increased yield of persons living with HIV was recorded. A total of 3,418 enrollees (males: 865, females: 2553) tested HIV positive and have been linked to treatment. The subsets of HIV positive enrollees include 753 OVC (males: 378, females: 375) and 2665 Caregivers (males: 487, females:2,178).

Conclusion Implementation of 'Assisted Referrals' and 'Incentivized Enrollment' on treatment, will increase access to HTS, linkage and retention on treatment and adherence for viral suppression, thereby contributing to UNAIDS 95-95-95 Goal. Absolute achievement was recorded on this project, with all the 43,495 enrollees knowing their HIV status and 3,418 that tested positive placed on treatment.

Disclosure No significant relationships.

\section{P210 ALINITY M HIV-1 ASSAY: DESIGN AND PERFORMANCE}

Jeffrey Wuitschick*, Anna Sobol, John Karavitis, John Salituro, Hemalata Joshi, Mark Sasaki, Tomasz Krupinski, Shihai Huang. Abbott Molecular, Research and Development, Des Plaines, USA

\subsection{6/sextrans-2019-sti.357}

Background HIV is characterized by a high degree of genetic diversity, presenting a challenge for the development of assays for initial diagnosis and subsequent monitoring of therapy response. Alinity $\mathrm{m}$ HIV-1 was developed on the Alinity $\mathrm{m}$ System, a fully automated, random/continuous access analyzer, to achieve accurate quantitation across groups, subtypes and circulating recombinant forms (CRF), concurrent HIV-1 confirmation and viral load monitoring in plasma, confirmation in serum.

Methods Abbott's Global Surveillance program was utilized to identify the most conserved target regions across HIV-1 variants. The assay targets two HIV-1 genomic regions and utilizes partially double-stranded probes, RNA-specific sample preparation chemistry, unit-dose lyophilized amplification reagents, and patented ReadiFlex ${ }^{\mathrm{TM}}$ sample processing logistics to deliver a time-to-first-result of 115 minutes. The Alinity $\mathrm{m}$ HIV-1 assay was evaluated for key performance attibutes.

Results Alinity m HIV-1 demonstrated linearity from 10 to 20,000,000 Copies/mL and demonstrated a within-laboratory $\mathrm{SD}$ of $\leq 0.13 \log$ Copies $/ \mathrm{mL}$ from 2.3 to $7.4 \log$ Copies $/ \mathrm{mL}$. Probit analysis demonstrated that the assay detected HIV-1 RNA with $95 \%$ probability at 13.88 Copies/mL using $3 \mathrm{rd}$ WHO HIV-1 Standard (subtype B). The assay exhibited $\geq 95 \%$ detection for HIV-1 group $\mathrm{M}$ subtypes, groups $\mathrm{O}$ and $\mathrm{N}$ at 20 Copies/mL. Correlation between Alinity m HIV-1 and Abbott RealTime HIV-1 assays demonstrated a mean bias of 0.03 Log Copies $/ \mathrm{mL}$ (95\% CI: -0.05 to 0.00 ). Confirmatory method agreement between Alinity m HIV-1 assay and comparator HIV-1 assay was 100\%.

Conclusion The Alinity m HIV-1 assay utilizes a state of the art instrument system and dual-target assay design to deliver highly sensitive detection of diverse HIV-1 groups/subtypes and accurate quantitation across a wide dynamic range while facilitating rapid turnaround time (115 minutes) and workflow flexibility. By providing confirmation and baseline viral load measurement in one test, the assay reduces the number of steps required for initial diagnosis of infection.

Disclosure No significant relationships.

\section{P211 REPRODUCTIVE OUTCOME AND FETAL GROWTH IN HIV- INFECTED PREGNANT WOMEN AT A UNIVERSITY HOSPITAL IN VITÓRIA, BRAZIL}

${ }^{1}$ Helena Lucia Barroso Helena Reis, ${ }^{2}$ Vinicius Barros, ${ }^{3}$ Ana Fernanda Rangel, ${ }^{4}$ Daniel Ribeiro Da Rocha, ${ }^{5}$ Paulo Roberto Merçon De Vargas, ${ }^{6}$ Angelica Miranda*. ${ }^{1}$ Federal University of Espirito Santo, Vitória, Brazil; ${ }^{2}$ Helena Lucia Barroso Helena Reis, Vitória, Brazil; ${ }^{3}$ Federal University of Espirito Santo, Vitória, Brazil; ${ }^{4}$ Federal University of Espirito Santo, Vitória, Brazil; ${ }^{5}$ Federal University of Espirito Santo, Pathology, Vitória, Brazil; ${ }^{6}$ Universidade Federal do Espirito Santo, Departamento de Medicina Social, Vitória, Brazil

\subsection{6/sextrans-2019-sti.358}

Background The infection by the human immunodeficiency virus (HIV), as well as the acquired immune deficiency syndrome (Aids), a worldwide epidemic, may lead to serious consequences in terms of maternal and fetal morbidity and mortality. The objective of this study was to describe the reproductive outcome and fetal growth in HIV-infected pregnant women and verify its relation to the antiretroviral use and severity of HIV infection.

Methods Cross-sectional study, with 122 pregnant women infected by HIV who had their termination in a university hospital maternity in Vitória, state of Espírito Santo, Brazil, from November 2001 to November 2014. The data was extracted from medical and public records in regard to gestational age, HIV status, antiretroviral use, and fetal dimensions. 